\title{
Design interface circuits for thick film heater for a gas sensor resistor
}

\begin{abstract}
In this paper an auto calibrated system of thick film resistive heater have been designed which can be adjusted to desired temperature. The circuit is consists of ADC, DAC, microcontroller, and current buffer. This circuit compensates dropt voltage across the heater which has been resulted from ambient temperature changes. Finally this circuit is able to control and adjust the heater's temperature automatically and also drives different resistive heaters for similar gas sensors.
\end{abstract}

Keyword: Auto calibration; Thick film resistive; Control heater; Ambient temperature; Compensating temperature 